\title{
BPM, Agile, and Virtualization Combine to Create Effective Solutions
}

\author{
Steve Kruba \\ Northrop Grumman \\ 15010 Conference Center Drive \\ Chantilly, VA, USA \\ email: steve.kruba@ngc.com
}

\author{
Steven Baynes \\ Northrop Grumman \\ 15010 Conference Center Drive \\ Chantilly, VA, USA \\ email: steve.baynes@ngc.com
}

\author{
Robert Hyer \\ Northrop Grumman \\ 15010 Conference Center Drive \\ Chantilly, VA, USA \\ email: bob.hyer@ngc.com
}

\begin{abstract}
The rate of change in business and government is accelerating. A number of techniques for addressing that change have emerged independently to provide for automated solutions in this environment. This paper will examine three of the most popular of these technologiesbusiness process management, the agile software development movement, and infrastructure virtualizationto expose the commonalities in these approaches and how, when used together, their combined effect results in rapidly deployed, more successful solutions.
\end{abstract}

Keywords-Agile; BPM; business process management; Rapid Solutions Development; Virtualization; Workflow

\section{INTRODUCTION}

Supporting change in today's dynamic environment requires a strategy and tools that can adapt to unforeseen events. Such tools have evolved in three key areas independently in response to this pressure.

Business Process Management (BPM) is both a management discipline and a set of technologies aimed at automating organizations' key business processes. Agility is a key feature of the products that support this market.

Agile Software Development is an approach for creating custom software and is designed to overcome some of the short-comings of more traditional approaches such as the waterfall methodology. It achieves agility through an iterative development approach that focuses on producing working software as quickly as possible.

Infrastructure Virtualization has expanded from server virtualization to storage, network, and desktop virtualization. The emphasis is on providing computing resources transparently to users and applications so that solutions can be stood up and modified quickly, and managed more easily and effectively.

The term agile has become popular for describing an important feature of modern information technology architectures. Agile within the context of each of the three technologies described in this article has a slightly different connotation, but the underlying principle remains the same. We will examine these similarities as well as the differences.

We will examine each of these approaches separately within their agile context and will discuss how in combina- tion they are becoming increasingly important to creating successful solutions. Examples from our experiences with our Northrop Grumman e.POWER ${ }^{\circledR 1}$ BPM product will be used to illustrate some of these ideas.

\section{Solutions}

When acquiring new software tools, organizations typically begin by examining the feature set of various products to determine which one is "best" at satisfying a set of requirements. We can lose sight of the fact that what we're really looking for is a solution to a problem-not the tool itself.

This might seem like either an obvious or a nonsensical statement, depending on how you look at it. Hasn't that always been the case with software development? you might ask. But the fact of the matter is that deploying systems has gotten more complicated in recent years. Quality issues, security issues, and compatibility issues have been given increased visibility as organizations have been "burned" by not appreciating their importance.

The effort involved in deploying finished solutions has become a significant part of the solution creation process. Deployment costs can be comparable to development cost when using model-driven tools in the BPM space. If those deployment (and support) costs can be reduced through technologies such as virtualization, that is significant.

Combining an effective software development tool with a powerful methodology like the agile process can be very beneficial. But writing software is probably not the best approach if there are solutions available that satisfy the requirements with pre-written software.

And finally, when we step back to thing about "solutions," we begin to focus on the effectiveness of the solutions produced. Key benefits for BPM and the agile methodology are on how well the solutions produced meet the actual needs of their stakeholders. Very often with complex systems requirements, asking stakeholders to define what they need is problematic because they simply do not have the experience to articulate the details. Both BPM and agile are specifically designed to reduce the risk of producing well-constructed solutions that are not effective at satisfying the true requirements;

\footnotetext{
${ }^{1}$ e.POWER has been providing solutions for government and commercial customers for over fifteen years and is a registered trademark of the Northrop Grumman Corporation.
} 

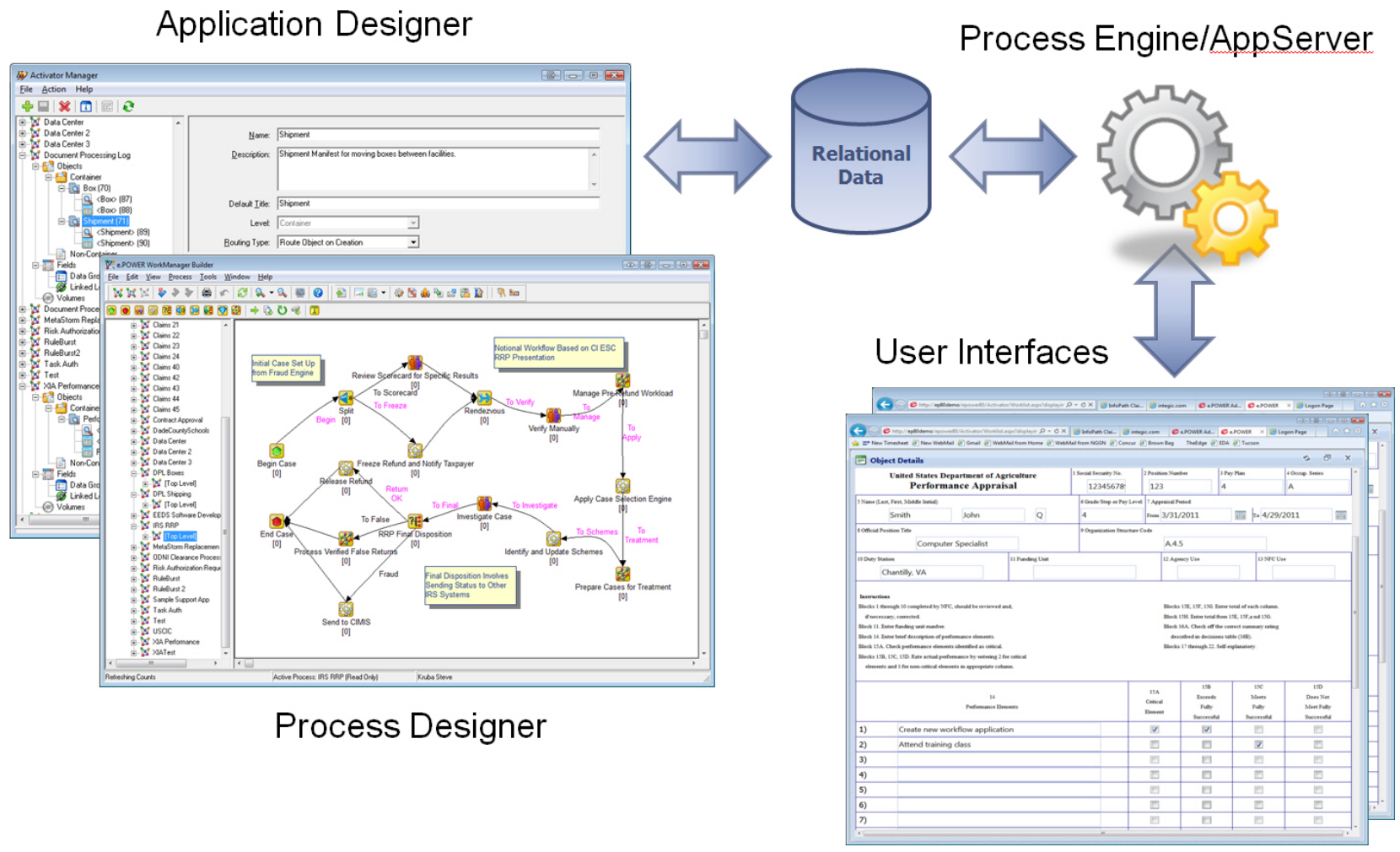

Figure 1. Model-Driven Architecture

i.e., producing a good solution that is not the right solution.

It's worth noting that a significant percentage of business solutions today involve some level of business process automation. Unlike other middleware components, rather than being just another tool used in constructing the solution, BPM software orchestrates the entire solution, even though it typically has to interact with many other infrastructure components (e.g., other applications and services) that satisfy important solution requirements.

In the next sections, we'll examine the three technologies in more detail. We will repeat the key theme of how they improve the agility of the overall solution in the generic sense (as opposed to the "agile software" sense) and hopefully gain insights into how to view these engagements from an overall solution perspective.

\section{Business Process Management}

Business process management, or BPM, is a management discipline typically supported by technology.[3] The purpose of BPM is process improvement. Software tools provide the technology base under which these goals are achieved. A typical BPM solution is composed of tasks performed by people and tasks performed by automated agents.

The BPM market, represented by over 100 vendors, is one of the fastest growing software segments per Gartner Dataquest while business process improvement has been ranked number 1 for the past five years by CIO's in the annual Gartner CIO survey.[4]

BPMS's such as e.POWER provide design environments that partition the work so that users with diverse skill-sets can work independently when developing a solution. Business users and business analysts play a major role in defining the business process and associated rules and can use graphical interfaces for defining these components. Graphics artists, rather than developers, can be used to design and implement the layout of user interfaces. Software developers create customizations that access legacy data from related applications, enhance the user interface by extending the out-of-the-box functionality when necessary, and extend the functionality of the process engine through exposed interfaces.

A key differentiator of BPMS's is that they are modeldriven. Other toolsets servicing the ВРM market and other business software segments are either parameterized, configuration-driven, or require writing custom software for the majority of the functionality. The difference is that BPMS's provide this functionality out-of-the-box.[5]. Parameterized or configuration-driven products are similar, but the connection between the production instantiation of the model is not as direct as model-driven products and only offer options that were pre-conceived by the product developers. Model-driven products offer much greater flexibility. ${ }^{2}$

\footnotetext{
${ }^{2}$ Gartner has written a lot on this topic.[2] [7] [8]
} 


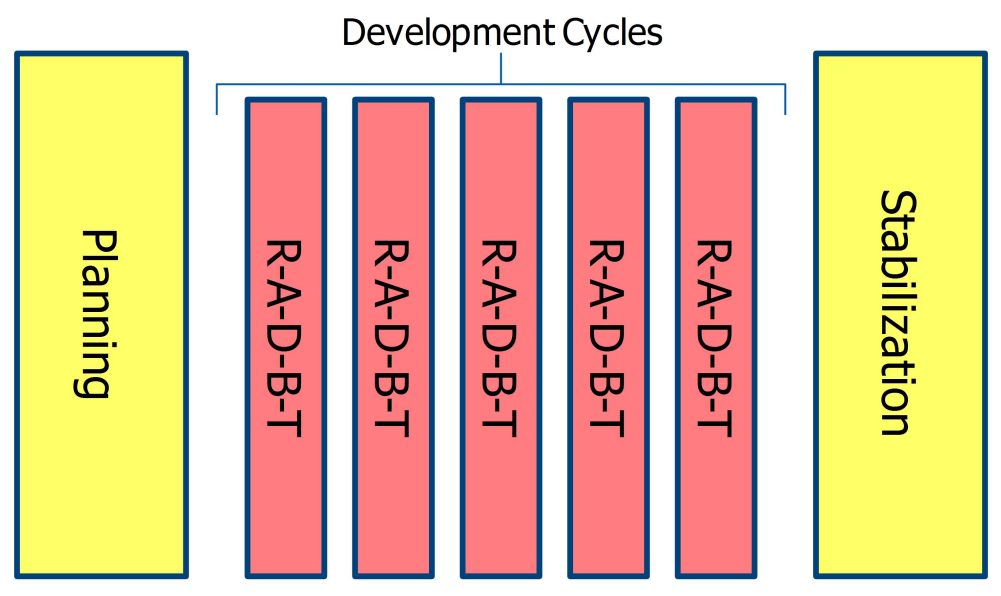

Requirements, Analysis, Design, Build, Test

Figure 2. Our Agile/Iterative Approach

A pictorial representation of model-driven BPM is shown in Figure 1. A graphical tool is used to define the business process, the results of which are stored in a backend repository - often a relational database. Likewise an application designer is used to define an application that is "process aware." This information is used by the process engine for enforcing the business rules and routing rules and by applications servers that drive the user interfaces. This same information is also available to end-users as they interact with the system for managing and performing work.

Note that some model-driven tools are used to define, not just the business process, but also applications that are process-enabled - the right-hand-side of Figure 1. The userinterfaces needed to actually process work within the business process are an important part of the solution, and being able to generate those interfaces through configuration rather than coding is a very powerful capability.[5]

The combination of capabilities provided by BPMS's fundamentally changes the way solutions are constructed in this problem space.

A BPMS product is purpose-built to create BPM solutions. Within the BPMS framework, the features that are common to all process problems are built into the product so that architects using the products simply deploy these pre-built components, augmented by customized components needed to represent the uniqueness of each particular solution. Frameworks such as service component architectures (SCA) within a service oriented architecture (SOA) are synergistic with BPMS's for the customization components. BPMS's could be viewed as pre-compiled frameworks.

This solutions-orientation is designed for rapid deployment and increased effectiveness. Being able to construct these solutions quickly while using these expressive tools to create more effective solutions is a key benefit. Effectiveness is achieved by using the tools to improve requirements validation, design, and solution creation, while improving quality and reducing risk.[5]

As we will see in the next sections, this agility can be amplified by other components of the solution infrastructure.

\section{Agile Software DeVelopment}

This section is not intended to be a how-to guide on agile software development - there are a number of other excellent resources. We will, however, include a discussion of agile principles to see how they relate to agility the other two technologies, illustrated by our experience in developing our own product.

Agile software development is first and foremost an iterative methodology for producing quality software. Agile development is characterized by frequent engagement of all stakeholders, including customers, developers, quality personnel, and management. Agile software development employs multiple development cycles to produce robust, testable feature sets, followed by integrated system testing.

We especially like the following quotation on the goal of agile software development.[6] This emphasizes the fact that all projects are time-bound and helps to avoid the problem of scope-creep.

\section{At the end of a project we would rather have $\mathbf{8 0 \%}$ of the (most important) features $\mathbf{1 0 0 \%}$ done, than $100 \%$ of all features $80 \%$ done.}

\section{A. e.POWER Agile Development}

To provide insight into agile development, we thought we would describe our first-hand experience using agile development for product releases of the e.POWER product over the past six years. 


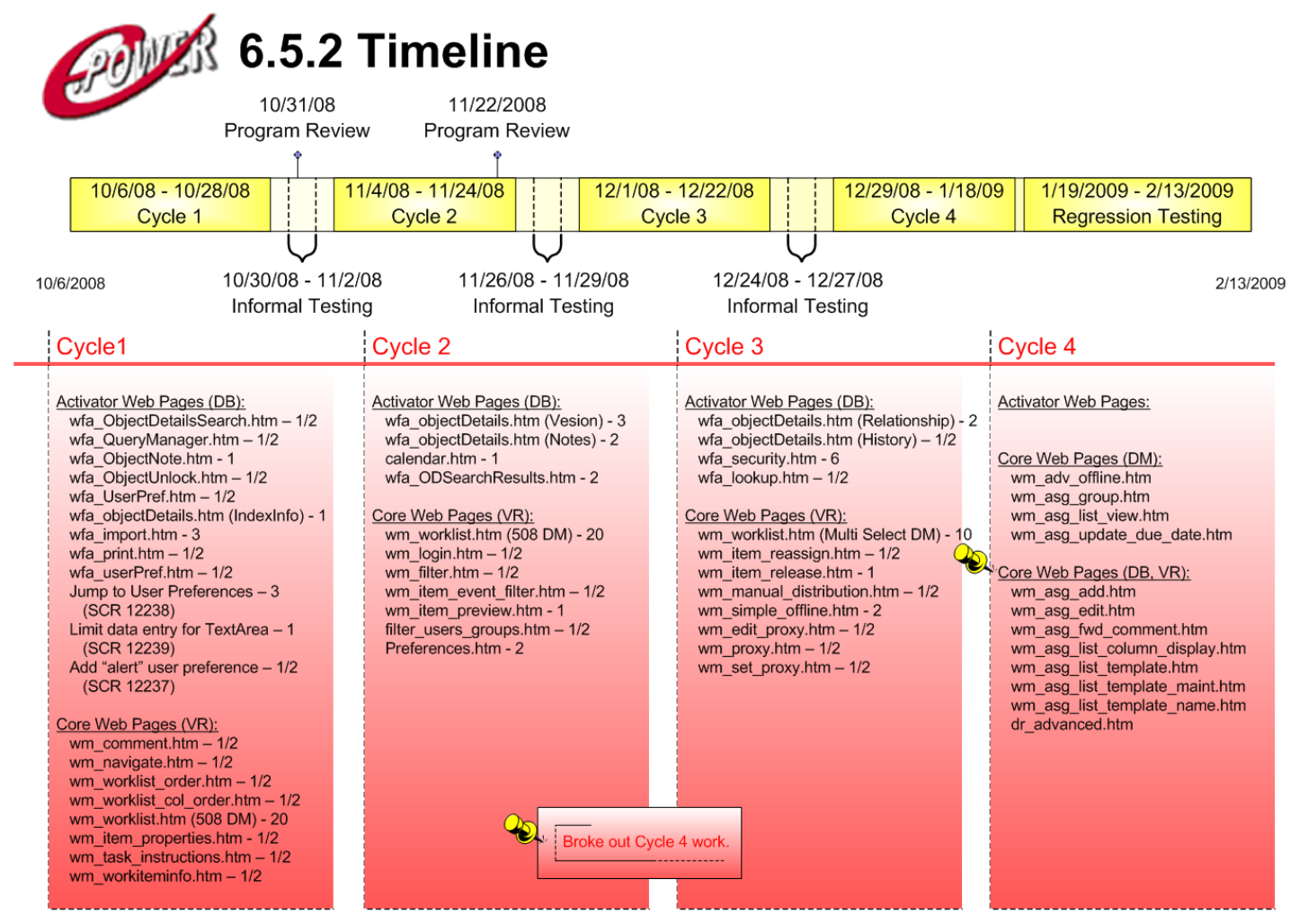

Figure 3. Release Timeline

Our approach consists of three phases for delivering a quality product: planning, multiple development cycles, and stabilization. Figure 2 illustrates this approach.

The planning phase begins with defining a vision statement and is followed by developing a list of features. Preliminary requirements are then collected and analyzed after which we can focus on the most important requirements first. Consistent with the agile manifesto[1], planning requires frequent involvement with our stakeholders. We kick off our planning sessions with a meeting with our advisory forum membership to be certain that we collect their high-level input as well as their detailed requirements.

After completing the planning phase, we are in a position to commit to what we will do in the iterative cycles. Each cycle is a mini-waterfall model, but much shorter, where we finalize requirements, perform analysis, design the software, build it, and test it. Every iteration or cycle contains a slice of the product, delivering small pieces of complete, working functionality.

The cycles provide opportunities for stakeholders that are not already part of the development cycles to review completed functionality. Since each cycle produces working software, demonstrations of that functionality are quite natural and simple to produce. These reviews also provide the opportunity to reprioritize the features and requirements list between cycles, since everyone is now more engaged and aware of the evolving solution.

Figure 3 provides an example of a release timeline of a past release of the e.POWER product. We have used this template for several years to manage the process. This one page summary of each release has been very effective at providing management, developers, and testers with visibility into the process and managing to the schedule.

After completion of the final cycle, we enter the last, or stabilization phase. At this point we perform complete system regression testing. Since we support multiple platforms for each release, we do platform testing during this phase. The configuration control board reviews the final requirements against our solution and documentation can now be finalized. Our quality manager is responsible for leading our final "total product readiness"3 process, which authorizes the product for commercial release.

\section{B. Relationship of Agile to BPM Solution Creation}

Our experiences with agile development of our software product may be interesting, but how does that relate to customers creating BPM solutions? They are related in two ways:

\footnotetext{
${ }^{3}$ Software products are comprised of much more than just software. Total product readiness is a term that we use to include the full breadth of capabilities that must be delivered for a product release, including marketing collaterals, release announcement materials, installation scripts, on-line help, documentation, training materials, etc.
} 
(1 BPM solutions typically involve writing some custom software. To the extent this is minimal, the more robust and effective the solution can be.[5] But when significant customization is required, an iterative agile approach can be beneficial.

2 Perhaps more importantly, the methodology used in writing software for agile software development is very similar to the iterative approach that we have used over the years in creating e.POWER solutions, including the model-driven aspects of the solution. The difference relates to code creation vs. solution creation. For agile BPM we are able to reduce the need to write custom software, replacing it with model manipulation-a nonprogramming effort.

\section{ViRTUALIZATION}

Virtualization is a much over-hyped technology, but not without some justification. Data centers world-wide are moving to virtualization to simplify operations, reduce hardware costs, reduce cooling and energy costs, and expedite solution deployments.

Although virtualization gained recognition initially with data center servers and has been in common usage for many years, virtualization has experienced much increased popularity recently in the areas of storage, networking, and desktops.

The following sections provide a high-level summary of the important subtopics on virtualization so that we can relate virtualization to our overall theme.

\section{A. Hardware}

Hardware virtualization abstracts the physical computer hardware from the operating system, allowing applications not originally designed for that combination to run on the new, virtualized platform.

Improved management features greatly reduce the manpower needed to configure, secure, backup, and operate virtualized servers than their physical counterparts. Furthermore, server virtualization solutions form the basis for cloud computing, which can be viewed as virtualization on steroids. Private clouds are virtualization platforms with even richer management features. The importance to our discussion is that adding new solutions to those environments becomes even easier yet, reducing operating costs.

Key aspects of virtual servers include higher availability (virtualization hardware can bring up offline copies of the server automatically), faster provisioning of new servers, automatic provisioning of new servers based on templates and security access rights, and much simpler hardware upgrades since the virtual machine is independent of the hardware. These are all aspects of agility that are important to our topic.

\section{B. Storage}

In a manner similar to hardware virtualization, storage virtualization abstracts the physical computer storage from the logical storage referenced in applications through the oper- ating system. The original impetus for storage virtualization may have been hardware independence - the desire to be able to swap out one vendor's disk drives for another vendor's when the old technology became obsolete. In general, features such as vendor independence, over-provisioning, replication, pooling, improved utilization, snapshots, etc., so significantly reduce operating costs that they typically offset any concern for a slight reduction in performance.

\section{Network}

Network virtualization uses software so that reconfiguring the physical network is not necessary to implement operational changes. The major benefit of network virtualization is simplified management of the network infrastructure. As new solutions are deployed, as new hardware is fielded, and as work-patterns evolve to support changing business requirements, network administrators can modify network configurations more easily than in the past.

\section{Desktop}

Desktop or client virtualization breaks the connection between users and their physical desktop machines. Multiple users share instances of servers for their desktop computing needs. Users require physical devices such as keyboards and monitors to interact with their virtual desktop servers, but these devices can be components of a system running a different operating system, or a purpose-built device limited to user interaction. In either case, an individual user can be granted access to one or more virtual desktops for specific work-tasks.

For some business cases, desktop virtualization offers significant benefits, but is not necessarily optimal for all use cases. When appropriate, desktop virtualization offers simpler and faster provisioning of new desktops, simplified desktop management in areas such as backups and patch management, better security, and improved reliability,

\section{E. Performance Issues}

A discussion of virtualization would not be complete without consideration of the performance implications. Virtualization provides an extra layer between applications and the physical hardware resources that has an associated cost. Our discussions will center around server virtualization.

For server virtualization, the hypervisor or Virtual Machine Monitor allows multiple operating systems to run concurrently on the machine hardware.

Type 1 or bare-metal hypervisors run directly on the host hardware while the virtual operating system(s) run on top of them. These tend to be more efficient than Type 2 hypervisors. Type 1 hypervisors include Microsoft Hyper-V, VMWare ESX and ESXi, and Citrix Xen Server.

Type 2 hypervisors run on top of a host operating system such as Microsoft Windows, adding an additional level between applications and the hardware. Type 2 hypervisors include VMWare workstation, VMWare server, and Microsoft Virtual Server.

The Virtual Insanity website has a useful graphic that illus- 


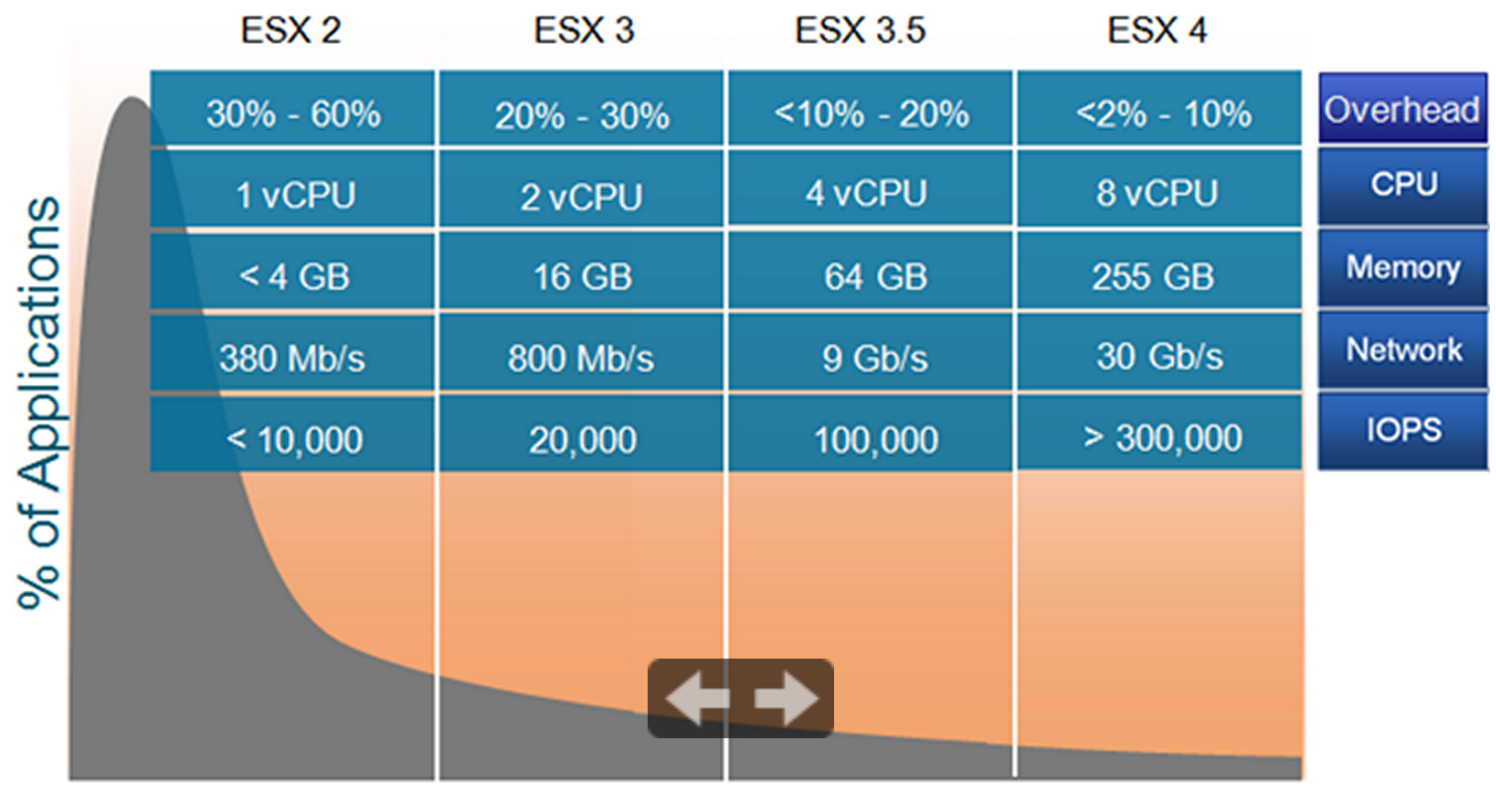

Figure 4. VMWare ESX Performance Improvements

trates the improvement in server virtualization performance in the VMWare ESX product - a Type 1 bare-metal hypervisor. As you can see from Figure 4, dramatic improvements have been made, reducing overhead from 30-60\% in ESX 2 to $2-10 \%$ in ESX 4.[9] Note corresponding improvements in network throughput and disk input/output (IOPS) as well. The point is that for mission critical systems, you need to benchmark your virtualized applications to insure adequate responsiveness and choose carefully to meet your needs.

Some Type 2 hypervisors are free and can be useful for some of your needs. For development and support of the e.POWER product, our primary need is to provide support for many old releases of the product, but since the activity levels are low, performance is not a critical issue. For production customers, we provide target memory and CPU's required to support designated workloads, and those targets are somewhat "diluted" when deployed in virtual environments. Depending on those activity levels, additional hardware may be needed, the cost of which may well be recouped in reduced operating and support costs, especially with the minimal overhead of the latest releases of Type 1 hypervisors.

On virtualized hardware, a key consideration is whether the storage is housed on internal drives or external storage. On platforms such as VMWare, complete filesystems are encapsulated when stored locally and are significantly less responsive than external storage such as SAN storage or NFS. Server virtualization platforms have special drivers for external storage that overcome this limitation.

\section{F. Our Experience}

We thought it would be valuable to include information on our experience with virtualization, primarily with hardware virtualization. The following chart summarizes the benefits we have seen from this transition over the past several years.

(1) Ability to setup virtual machines quickly and move them or turn them on or off as needed

2 Reduced hardware investment and on-going power costs

3 More efficient use of hardware resources

4 Reduced labor expenses in moving virtual machines to new hardware-no operating system reinstallation necessary

5 Features such as "snapshot" facilitates testing of installation scripts and configuration

6 Entire virtual machines are backed up as a single file

$(7$ Virtual machines are indistinguishable from physical machines from an end-user perspective

\section{COMMON THEMES}

So what are some of the common themes we see in the three technologies presented above? At a high level, they center around the concept of agility: providing the ability to create solutions that are both quick to produce and adaptable to needs that evolve over time. Speed is an important component but equally important is effectiveness, emphasizing the overall development process from needs assessment through deployment. The three approaches highlighted in this article are not the only ones that apply these principles, but are three of the most visible today and touch on all aspects of solutions in the business context. An organization that emphasized at least these three would be well served. 
Agility is about embracing change, knowing that user requirements will evolve as the emerging solution provides greater visibility into the final product. BPMS and agile toolsets make it possible to iterate towards a solution because of the flexibility they introduce into the creation process. We are often at a loss to express what the ultimate solution needs to look like, but we can more readily recognize it when we see it.

A high level summary of principles that are shared among these three approaches are as follows.

(1) People are the key to solutions. Technology has reached a level of refinement where we no longer should be optimizing bits and bytes, but optimizing people, including process participants, architects and developers, and support staff.

2 Engage stakeholders continuously throughout the solution development process. Continuous feedback with iterative evolution of the solution fundamentally improves the creation process.

(3) Working software is the best visualization tool for working software. Modern software has become increasingly expressive, for which there is no substitute.

4 Task the right people for each aspect of solution creation based on domain knowledge. The tools now allow business people to participate in this process, allowing information technology professionals to focus on the ITaspects of solutions.

(5 Modularity is a theme seen in all three of these technologies, allowing participants to easily conceptualize the current component of interest.

6 Virtualization makes infrastructure less of an impediment to productivity. People can more easily gain access to infrastructure resources in creating and managing solutions.

\section{CONCLUSIONS}

Creating successful automated solutions is challenging in today's highly competitive environment. Solutions must be conceived and implemented quickly in a manner that allows them to adapt as needs change. For a large class of business problems, this requires the capabilities of a business process management suite. BPMS's differentiate themselves by their rapid solutions creation capabilities achieved through a model-driven architecture. Since significant BPM projects require some custom software for critical parts of the solution, agile principles are very compatible with BPMS's and Northrop Grumman's e.POWER product software is developed using an agile software development methodology.

Creating or evolving a solution rapidly is of little consequence if it cannot be fielded in a like manner and this is where virtualization becomes important. The underlying underpinnings of agility in each of these aspects of solution creation work together to insure that solutions are effective and deployed in a timeframe that meets the needs of their business customers.

\section{REFERENCES}

[1] http://agilemanifesto.org/principles.html, 2001.

[2] M. Blechar. The changing concept of model-driven approaches. Gartner, pages 1-8, August 2009.

[3] Michelle Cantara. BPM research index: Business process management technologies. Gartner, pages 1-11, September 2009.

[4] Gartner EXP worldwide survey of nearly 1,600 CIOs shows IT budgets in 2010 to be at 2005 levels. http://www.gartner.com/ it/page.jsp ?id=1283413, January 2010.

[5] S. Kruba. Significance of rapid solutions development to business process management. International Journal of Computer Science and Information Security, 8(4):299303, 2010.

[6] Malotaux. Evolutionary project management methods. http://www.malotaux.nl/doc.php?id=1, August 2007.

[7] D. Plummer and J. Hill. Three types of model-driven composition: What's lost in translation? Gartner, pages 1-10, August 2008.

[8] D. Plummer and J. Hill. Composition and BPM will change the game for business system design. Gartner, pages 1-21, December 2009.

[9] Life as a VMWare virtual machine, 2010. http://www.virtualinsanity.com /index.php /2010/08/17 /life-as-a-vmware-virtual-machine/.

\section{AUTHOR PROFILES}

Steve Kruba is chief technologist for Northrop Grummans process-oriented commercial software products, including e.POWER, and a Northrop Grumman Technical Fellow. Steve has 42 years of experience developing software and solutions for customers. He holds a Bachelor of Arts in Mathematics and a Master of Science in Management Sciences from the Johns Hopkins University.

Steve Baynes is the department manager for the e.POWER product development team with extensive agile experience. $\mathrm{He}$ is a certified ScrumMaster, member of the Agile Alliance organization and speaks often on Agile development. As the manager for the e.POWER product, Steve works with business development, project implementation teams, and customers to continually improve the e.POWER product from both a feature and quality perspective.

Bob Hyer is chief architect for the e.POWER product development team. Bob has over 30 years of experience developing software solutions and software products for government and commercial customers. He has a Bachelors of Science in Business Management from Virginia Tech and a Master of Science in Technology Management from American University. 